\title{
Monitoring the censored lognormal reliability data in a three-stage process using AFT model
}

\author{
Azam Goodarzi $^{1} \cdot$ Amirhossein Amiri $^{1} \cdot$ Shervin Asadzadeh $^{2}$
}

Received: 8 January 2016/Accepted: 29 August 2016/Published online: 8 September 2016

(c) The Author(s) 2016. This article is published with open access at Springerlink.com

\begin{abstract}
Improving the product reliability is the main concern in both manufacturing and service processes which is obtained by monitoring the reliability-related quality characteristics. Nowadays, products or services are the result of processes with dependent stages referred to as multistage processes. In these processes, the quality characteristic in each stage is affected by the quality characteristic in the previous stages known as cascade property. Two regression-adjusted control schemes are applied to monitor the output quality variables of interest. Moreover, censoring is among the main limitations while monitoring the reliability-related quality characteristics, causing not to record the real values of some observations. Hence, the right censored observations are used to extend monitoring schemes under both the fixed- and variable-competing risks. In this paper, the accelerated failure time (AFT) is used to relate the reliability-related quality characteristic with lognormal distribution to the incoming variables. Then, two cause-selecting control charts are developed to monitor outgoing quality variables when censoring happens in each reliability-related stage. The performance of the control charts is evaluated and compared through
\end{abstract}

Amirhossein Amiri

amiri@shahed.ac.ir

Azam Goodarzi

a.goodarzi@shahed.ac.ir

Shervin Asadzadeh

sh_asadzadeh@iau-tnb.ac.ir

1 Department of Industrial Engineering, Faculty of Engineering, Shahed University, Tehran, Iran

2 Department of Industrial Engineering, Islamic Azad University, North Tehran Branch, Tehran, Iran extensive simulation studies under the censored and noncensored scenarios.

Keywords Accelerated failure time (AFT) model . Multistage process - Cascade property $\cdot$ Regressionadjusted control schemes - Fixed- and variable-competing risks

\section{Introduction}

Manufacturing or service processes do not solely include one-stage processes, and the products' reliability is mostly the result of several different process steps. In such multistage processes, cascade property is a principal feature in which the outgoing quality characteristic is affected by the changes in the incoming variables (Yang 1999; Tsung et al. 2008). To deal with this problem, cause-selecting chart (CSC) is first introduced by Zhang (1984). The CSCs are most widely used to monitor and diagnose the multistage processes. Two-stage processes with normal output variable are reviewed by Wade and Woodall (1993). The CSC can also be used to monitor more than two-stage processes (Xiang and Tsung 2008). However, there exists a critical issue that quality variables do not follow the normal distribution in all the cases, while the major assumption using the CSC monitoring procedure is normally distributed quality characteristic. Therefore, some other regression-adjusted monitoring procedures based on the generalized linear models (GLMs) have been proposed to relax the normality assumption. These models have been extended to include the exponential family distributions (Jearkpaporn et al. 2003, 2007; Skinner et al. 2003).

To improve the product reliability in manufacturing and service processes, monitoring and evaluating reliability 
data are widely used. The reliability data have two main features which make the monitoring of such data complicated. The first one is that the reliability data often follow the specific parametric distributions, namely, location scale and log-location scale (Meeker and Escobar 1998). Among them, the lognormal, Weibull, and extreme value are the most commonly used distributions to model reliability data (Lawless 2003). The second issue is that it is not always possible to record the exact values of the reliability data due to the lack of time, resources or expenses. This problem makes us to consider the censoring as another property while dealing with reliability data. Furthermore, it would be more complicated when the variable-competing risk takes place.

Many authors investigated the monitoring of the reliability data in the literature. Padgett and Spurrier (1990) developed Shewhart-type chart to detect the shift of a percentile of lognormal and Weibull reliability data. Xie et al. (2002) proposed a new technique to monitor the interfailure times which follow the exponential distribution. After that, the control charts were extended to monitor time between $r$ failures (Surucu and Sazak 2009). In addition, Zhang et al. (2011a, b) designed the economic inter-failure times control chart system when the times follow the exponential distribution. Nichols and Padgett (2006) designed a new bootstrap control chart for monitoring the tensile strength of carbon fiber. Batson et al. (2006) suggested a new approach to monitor mean time between failures. This approach is based on transforming exponential, lognormal, and Weibull failure time data to normal distributed data. The CUSUM control chart has been developed to monitor the time between events by Shafae et al. (2014). In addition, Akhundjanov and Pascual (2015) proposed the EWMA control chart to diagnose the small shifts when a single measurement is taken per sampling period.

In real situations, the observations may be censored at a pre-specified level because of time and cost limitations. Moreover, the variable-competing risk may be considered as well which censors the values of response quality variables. For example, it is not possible to record the values of bond strength accurately when the tensile strength of adhesive bond between vinyl fabric and PVC foam backing is monitored and evaluated. This problem would happen due to the failing of the foam backing sooner. Then, this kind of censoring is introduced as a variable-competing risk which cannot be easily neglected for the sake of proposing optimal monitoring schemes. It is significant that the main assumption of all the above-mentioned researches is that the observations were recorded with no fixed and variable censoring, even though the location-scale and loglocation-scale distributions have been addressed. Censored observations at fixed level are considered for monitoring in
Steiner and MacKay (2000). In addition, Steiner and MacKay (2001) proposed exponentially weighted moving average (EWMA) control chart based on the conditional expected value to monitor the bond strength values which are censored under the competing risk variable. Moreover, the EWMA control chart is used to monitor the mean of censored lifetimes when censoring occurs at fixed level (Zhang and Chen 2004; Lu and Tsai 2008). Then, some control charts are developed to monitor the time-to-failure data in the presence of right censoring using rank tests ( $\mathrm{Li}$ et al. 2012; Li and Kong 2015). The base of these control charts is using the rank statistics to derive the generic formula for the operating characteristic functions of the control chart.

On the other hand, as discussed earlier, the products usually come from processes with more than two stages. Shamsuzzaman et al. (2009) developed a control chart system to monitor the time between successive events at different process stages in the multistage manufacturing system. Asadzadeh and Aghaie (2012) proposed two control charts to monitor and improve the product reliability in a two-stage process with this assumption that the reliability data follow the Weibull distribution. Moreover, the outgoing quality characteristic is censored due to the presence of both the fixed- and variable-competing risks. In addition, Asadzadeh et al. (2013) proposed two regression-adjusted control schemes based on Cox-Snell residuals. In their paper, the control charts were extended to monitor the residuals in the presence of the right censoring at fixed level. In these papers, the AFT model is used to relate the quality characteristics. Furthermore, Zhang et al. (2015) investigated the effect of estimation error on the performance of risk-adjusted survival time CUSUM scheme in continuous time with the cardiac surgery data. They used the AFT model in their research that can be applied in multistage processes. Asadzadeh et al. (2014a, b) used the Proportional Hazard and Frailty models to relate the quality characteristics in a two-stage process. The monitoring schemes were developed in the presence of the right censoring at fixed level. In addition, Zhou et al. (2012) used proportional hazards $(\mathrm{PH})$ model to link upstream supply chain quality/testing information as explanatory covariates for the early detection of reliability problems which is similar to the $\mathrm{PH}$ model for the multistage processes and cascade property.

It should be noted that all the discussed processes are assumed to have two stages and the outgoing quality characteristic has been censored. However, there exists a real case dealing with cotton spinning in which the three quality characteristics are Fiber Length, Fiber Strength, and Skein Strength. If the fiber length was to change, it would lead to change in the fiber strength. Moreover, a change in any of the two quality variables would affect the skein 
strength of the spun cotton. Obviously, fiber strength and skein strength are the reliability-related quality characteristics which bring about censored observations for the second and third stages. Considering the mentioned example and the concept of variable-competing risk discussed in the case of adhesive bond between vinyl fabric and foam backing, we address a three-stage process in which the quality characteristics of both the second and third stages evaluate and reflect the reliability of product. Thus, we use the residuals to construct the CSC and monitor the outgoing quality characteristic. It is remarkable that the proposed monitoring schemes are extended when the censoring occurred in these stages. As mentioned before, the lognormal distribution is widely used to model the reliability data. Furthermore, it is assumed that these reliability-related quality characteristics follow the lognormal distribution.

The structure of this paper is as follows: the problem statement and the AFT model are elaborated in the next section. The monitoring schemes under the absence and presence of the fixed- and variable-competing risks are proposed in Sect. 3. In Sect. 4, the performance of the proposed control charts is studied and compared by extensive simulation studies. Numerical example is provided in Sect. 5. Concluding remarks are presented in Sect. 6.

\section{Problem statement}

As illustrated in Fig. 1, suppose a three-stage manufacturing process in which the outgoing quality characteristic is affected by quality variables of the previous stages. In this process, the quality characteristics in the second and third stages reflect the reliability of product which are denoted by $y_{1}$ and $y_{2}$, respectively. Thus, censoring would occur at fixed level due to the cost and time constraints in any of the discussed stages. Besides, some of actual observations would be censored due to the presence of another censoring mechanism which is referred to as variable-competing risk. As a result, two distinct competing risks, namely, fixed and variable, would happen in the process which prohibits the exact records of some values corresponding to the reliability-related quality characteristics of the second and third stages.

Taking into account the dependency of reliability data to the previous stages, the survival analysis regression models have been used. There are two major approaches in this

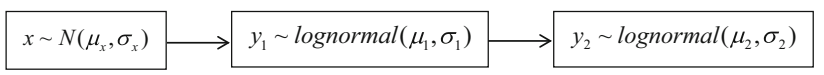

Fig. 1 Three-stage process case. One is the accelerated failure time (AFT) model which relates the parameters of output quality characteristic to the identified covariates and the other one is the proportional hazards $(\mathrm{PH})$ models in which covariates affect the associated hazard function (Padgett and Spurrier 1990). Since the AFT model is the most widely used models among the parametric ones, in this paper, the AFT model is considered to relate the parameter of the reliability-related quality characteristics of both the third and second stages to the quality characteristics in the previous stages.

As discussed in the previous section, we assumed that the reliability-related quality characteristics of both the third and second stages follow the lognormal distribution due to extensive usages among the variety distributions which can be used in survival regression models. Therefore, the general probability density and survival function of the lognormal distribution are given in Eqs. (1) and (2), respectively, as follows:

$f(y)=\frac{1}{\sqrt{2 \pi} \sigma y} e^{-\left(\frac{1}{2}\left(\frac{\log y-\mu}{\sigma}\right)^{2}\right)} \quad y>0$,

and

$s(y)=1-\phi\left(\frac{\log y-\mu}{\sigma}\right)$.

For the parameterization, $\mu$ is the location parameter and $\sigma$ is the shape parameter. These parameters are the mean and standard deviation of the associated normal distribution, respectively. In addition, $\phi($.$) is the cumulative dis-$ tribution function of normal standard distribution. It is assumed in this paper that only the location parameters of the quality characteristics in the second and third stages $\left(\mu_{1}\right.$ and $\mu_{2}$ ) depend on covariates of the previous stages and their dependency is shown as follows:

$\mu_{y_{2} \mid x, y_{1}}=\alpha_{0}+\alpha_{1} x+\alpha_{2} \log \left(y_{1}\right)$,

and

$\mu_{y_{1} \mid x}=\beta_{0}+\beta_{1} x$.

Then, the AFT-based survival functions are defined for quality characteristics of both the third and second stages as

$s\left(y_{2} \mid x, y_{1}\right)=1-\phi\left(\frac{\log y_{2}-\alpha_{0}-\alpha_{1} x-\alpha_{2} \log y_{1}}{\sigma_{2}}\right)$,

and

$s\left(y_{1} \mid x\right)=1-\phi\left(\frac{\log y_{1}-\beta_{0}+\beta_{1} x}{\sigma_{1}}\right)$,

where $\beta_{0}, \beta_{1}, \alpha_{0}, \alpha_{1}$, and $\alpha_{2}$ are the parameters of regression models of two different reliability-related quality 
characteristics. In addition, $\mu_{1}, \sigma_{1}, \mu_{2}$, and $\sigma_{2}$ are considered as the mean and standard deviation of the quality characteristics in the second and third stages $\left(y_{1}\right.$ and $\left.y_{2}\right)$. Without loss of generality, the covariates most widely follow the normal distribution, i.e., $x \sim N\left(\mu_{x}, \sigma_{x}\right)$. Therefore, this fact directs us toward using $\log \left(y_{1}\right)$, which transforms the distribution of the second-stage quality characteristic to normal distribution, to relate the parameter of quality characteristic of the third stages to the previous stage.

In the next section, the cause-selecting control strategies are elaborated based on the AFT model. The two proposed control charts are extended in the presence of both the fixed- and variable-competing risks.

\section{Regression-adjusted control schemes}

In this section, the control charts are designed under two scenarios whether the process observations are censored or not. Subsequently, we have proposed the monitoring schemes in the following sub-sections for the sake of finding out-of-control situations immediately.

\section{Regression-adjusted control schemes in the absence of censoring}

In this sub-section, monitoring schemes are developed in the absence of both the fixed- and variable-competing risks. Doing so, we concentrate on developing the control charts to monitor the quality characteristics to detect decreasing shifts, although it is easy to establish the proposed control charts for the sake of detecting increasing and decreasing shifts simultaneously. Accordingly, two regression-adjusted control schemes, including CUSUM and EWMA, are proposed.

Having represented the manufacturing process and the corresponding distributions for quality characteristics, we concentrate on developing CUSUM control chart to monitor the response variable, first. The statistic of this proposed control chart is constructed based on the likelihood function. The likelihood function related to the $i$ th observation in the absence of censoring is modified as $L_{i}=f\left(y_{i}\right)$ that can be calculated by Eq. (1). Then, the one-sided CUSUM statistic is given by

$c_{i}^{-}=\min \left\{0, c_{i-1}^{-}-w_{i}\right\}$,

$c_{0}^{-}=0$,

where $w_{i}$ is the CUSUM score proposed by Page (1954) and is computed by

$w_{i}=\log \left(\frac{L\left(\omega_{i 1} \mid y_{2 i}\right)}{L\left(\omega_{i 0} \mid y_{2 i}\right)}\right)$.

In this equation, $\omega_{i 0}$ and $\omega_{i 1}$ are defined as in-control and out-of-control values of the parameters corresponding to the quality characteristic in the last stage. The vector of incontrol parameter is shown as $\omega_{i 0}=\left(\alpha_{0}+\alpha_{1} x+\alpha_{2}\right.$ $\left.\log \left(y_{1}\right), \sigma_{2}\right)$, and the out-of-control parameter vector is $\omega_{i 1}=\left(\alpha_{0}+\alpha_{1} x+\alpha_{2} \log \left(y_{1}\right)-\delta, \sigma_{2}\right)$, where $\delta$ is the decreasing shift in the in-control location parameter. Taking our attention into the mean of survival time which is calculated by following equation, it implies that changing the location parameter leads to changing the mean of survival time:

$E\left(y_{2}\right)=\exp \left(\mu_{2}+\frac{\sigma_{2}^{2}}{2}\right)$.

Hence, the CUSUM score is given by the following equation based on the lognormal density function of quality characteristic in the third stage:

$w_{i}=\frac{-\delta}{2 \sigma_{z}^{2}}\left(2 \log \left(y_{2 i}\right)\right)+\delta-2 \mu_{2 i}$.

This statistic is set to detect decreasing shifts, even though the CUSUM control procedure signals an out-ofcontrol status when its statistic exceeds the lower control limit, $\mathrm{LCL}_{1}$. The $\mathrm{LCL}_{1}$ is set by simulation to achieve the desired in-control average run length (ARL) criterion.

Since the proposed CUSUM control chart is CSC, the next monitoring surveillance EWMA control chart is also proposed based on the residuals to omit the effect of covariates in the previous stages on the quality characteristics in the last stage. The residuals are computed as follows:

$z_{i}=\frac{y_{2 i}-E\left(y_{2 i}\right)}{\sqrt{\operatorname{Var}\left(y_{2 i}\right)}}$,

where $E\left(y_{2 i}\right)$ and $\operatorname{Var}\left(y_{2 i}\right)$ are the mean and variance of the quality characteristic in the third stage. Since the quality characteristic of the last stage follows the lognormal distribution, the mean of survival time is given by Eq. (9) and its variance is calculated as follows:

$\operatorname{Var}\left(y_{2}\right)=\exp \left(2 \mu_{2}+\sigma_{2}^{2}\right)\left(\exp \left(\sigma_{2}^{2}\right)-1\right)$.

By considering Eq. (3), the mean and variance of lognormal quality characteristic in the third stage are calculated as $E\left(y_{2}\right)=e^{\alpha_{0}+\alpha_{1} x+\alpha_{2} \log \left(y_{1}\right)+\frac{\sigma_{2}^{2}}{2}}$ and $\operatorname{Var}\left(y_{2}\right)=$ $e^{2\left(\alpha_{0}+\alpha_{1} x+\alpha_{2} \log \left(y_{1}\right)\right)+\sigma_{2}^{2}}\left(e^{\sigma_{2}^{2}}-1\right)$. The proposed residual $z_{i}$ in Eq. (11) is standardized; hence, the mean and standard deviation of $z_{i}$ are equal to 0 and 1 , respectively. Note that changing the scale parameter of the last stage quality characteristic $\mu_{2}$ leads to changing in the mean of interest. Consequently, the one-sided EWMA statistic based on the residuals in Eq. (11), is derived as follows to detect decreasing shifts in the mean of the quality characteristic in the third stage: 


$$
\begin{aligned}
Q_{i} & =\min \left\{\mu_{0}, \lambda z_{i}+(1-\lambda) Q_{i-1}\right\} \\
& =\min \left\{0, \lambda z_{i}+(1-\lambda) Q_{i-1}\right\},
\end{aligned}
$$

where $\lambda$ is the smoothing parameter $(0<\lambda \leq 1)$ and $Q_{0}$ is equal to the in-control mean of the residual statistic, i.e., $Q_{0}=0$. The general one-sided lower control limit of the EWMA statistic is given by $\mathrm{LCL}_{2}=\mu_{0}-L \sigma \sqrt{\frac{\lambda}{2-\lambda}}$. Since $z_{i}$ are standardized residuals, then $\mathrm{LCL}_{2}$ reduces to

$$
\mathrm{LCL}_{2}=-L \sqrt{\frac{\lambda}{2-\lambda}} .
$$

The one-sided EWMA control chart triggers a signal when $Q_{i}<\mathrm{LCL}_{2}$. The $\mathrm{LCL}_{2}$ is set, such that a desirable incontrol ARL is obtained.

In the next sub-section, our proposed control charts are developed when the censoring data exist.

\section{Regression-adjusted control schemes in the presence of censoring}

After proposing the monitoring schemes in the absence of censoring mechanisms, we concentrate on developing the control chart strategies in the presence of both the fixed- and variable-competing risks. Moreover, we present the formulas regardless of which stage is considered. At the end, they would be adopted to the quality characteristics $y_{2}$ and $y_{1}$.

As mentioned before, the variable-competing risk can occur unwantedly which precludes the accurate recording of quality characteristic values. We assume that this variable follows the normal distribution. Therefore, the values of reliability-related quality characteristics are recorded completely unless they reach the pre-determined limit denoted by $c$ or the failure related to variable-competing risk, which is denoted by $c r$. Given that the existence of the fixed- and variable-competing risks affect the actual values deleteriously, all censored data can be replaced with their conditional expected values (CEV) which are calculated by the following formulas considering which competing risk occurs first
$w_{c}=\operatorname{CEV}(y \mid y>c) \quad$ or $\quad w_{c r}=\operatorname{CEV}(y \mid y>c r)$,

where the CEV weights of the process are defined as follows:

$w= \begin{cases}y & \text { if } y \leq c \text { and } y \leq c r \\ w_{\mathrm{c}} & \text { if } c<y \text { and } c \leq c r . \\ w_{\mathrm{cr}} & \text { otherwise }\end{cases}$

Assuming lognormal distribution, the CEV of the quality characteristics observation censored at $q$ is derived by the following equation regardless of which competing risk occurs

$\operatorname{CEV}(y)=E(y \mid y>q)=\frac{\frac{1}{2} e^{\frac{1}{2} \sigma^{2}+\mu}+\frac{1}{2} e^{\frac{1}{2} \sigma^{2}+\mu} \operatorname{erf}\left(\frac{\mu-\log y+\sigma^{2}}{\sqrt{2} \sigma}\right)}{S(q)}$,

where $S(q)$ is the survival function of the reliability-related quality characteristic, and erf(.) is the error function that is defined as follows:

$\operatorname{erf}(t)=\int_{0}^{t} \frac{2}{\sqrt{\pi}} e^{-t^{2}} \mathrm{~d} t$

Subsequently, the CEV weight, which was presented in Eq. (16), can be given by the following equation:

$w= \begin{cases}\frac{1}{\frac{1}{2} e^{\frac{1}{2} \sigma^{2}+\mu}+\frac{1}{2} e^{\frac{1}{2} \sigma^{2}+\mu} \operatorname{erf}\left(\frac{\mu-\log (c)+\sigma^{2}}{\sqrt{2} \sigma}\right)} & \text { if } \quad y \leq c \text { and } y \leq c r \\ \frac{\frac{1}{2} e^{\frac{1}{2} \sigma^{2}+\mu}+\frac{1}{2} e^{\frac{1}{2} \sigma^{2}+\mu} \operatorname{erf}\left(\frac{\mu-\log (c r)+\sigma^{2}}{\sqrt{2} \sigma}\right)}{S(c r)} & \text { if } c<y \text { and } c \leq c r \\ \frac{\text { otherwise. }}{}\end{cases}$

Now, we can establish the CEV weights for the reliability-related quality characteristic of the second stage by considering $y_{1}, \beta_{0}+\beta_{1} x$, and $\sigma_{1}$ instead of $y, \mu$ and $\sigma$, respectively, in Eq. (18). Thus, the CEV weights of the second stage can be indicated as follows:

$$
w_{1}= \begin{cases}\frac{y_{1}}{\frac{1}{2} e^{\frac{1}{2} \sigma_{1}^{2}+\beta_{0}+\beta_{1} x}+\frac{1}{2} e^{\frac{1}{2} \sigma_{1}^{2}+\beta_{0}+\beta_{1} x} \operatorname{erf}\left(\frac{\beta_{0}+\beta_{1} x-\log \left(c_{1}\right)+\sigma_{1}^{2}}{\sqrt{2} \sigma_{1}}\right)} & \text { if } \quad y_{1} \leq c_{1} \text { and } y_{1} \leq c r_{1} \\ \frac{S\left(c_{1}\right)}{2} & \text { if } \quad c_{1}<y_{1} \text { and } c_{1} \leq c r_{1} \\ \frac{\frac{1}{2} e^{\frac{1}{2} \sigma_{1}^{2}+\beta_{0}+\beta_{1} x}+\frac{1}{2} e^{\frac{1}{2} \sigma_{1}^{2}+\beta_{0}+\beta_{1} x} \operatorname{erf}\left(\frac{\beta_{0}+\beta_{1} x-\log \left(c r_{1}\right)+\sigma_{1}^{2}}{\sqrt{2} \sigma_{1}}\right)}{S\left(c r_{1}\right)} & \text { otherwise, }\end{cases}
$$


where $c_{1}$ and $c r_{1}$ are the pre-determined limit and the variablecompeting risk of the second-stage quality characteristic.

Constructing the CEV weights of the third-stage quality characteristics is similar to the second one, but there is a
The proposed likelihood function is used to construct the CUSUM score which is defined in Eq. (8).

As a consequence, the lognormal CUSUM score for the censored observations is written as

$w_{i}=\log \left(\frac{\left[\left(1-F\left(t_{i} \mid \omega_{i 1}\right)\right)\left(1-G\left(t_{i}\right)\right)\right]^{\tau_{i}} \times\left[\left[f\left(t_{i} \mid \omega_{i 1}\right)\left(1-G\left(t_{i}\right)\right)\right]^{\gamma_{i}}\left[g\left(t_{i}\right)\left(1-F\left(t_{i} \mid \omega_{i 1}\right)\right)\right]^{\left(1-\gamma_{i}\right)}\right]^{1-\tau_{i}}}{\left[\left(1-F\left(t_{i} \mid \omega_{i 0}\right)\right)\left(1-G\left(t_{i}\right)\right)\right]^{\tau_{i}} \times\left[\left[f\left(t_{i} \mid \omega_{i 0}\right)\left(1-G\left(t_{i}\right)\right)\right]^{\gamma_{i}}\left[g\left(t_{i}\right)\left(1-F\left(t_{i} \mid \omega_{i 0}\right)\right)\right]^{\left(1-\gamma_{i}\right)}\right]^{1-\tau_{i}}}\right)$.

difference in defining the location parameter, $\mu_{2}$. Since the censoring mechanism occurs in stage two as well, it is indispensable to use the CEV weights of the second stage for defining $\mu_{2}$ specified in Eq. (3). Consequently, the CEV weights of the third stage quality characteristic can be obtained as follows when the location parameter $\mu_{2}$ is defined as $\alpha_{0}+\alpha_{1} x+\alpha_{2} \log \left(w_{1}\right)$ :
At the end, this score is reduced to

$$
\begin{aligned}
w_{i}= & \tau_{i} \log \left[\frac{1-\Phi\left(\frac{\ln c-\mu_{0}}{\sigma_{y}}\right)}{1-\Phi\left(\frac{\ln c-\left(\mu_{0}-\delta\right)}{\sigma_{y}}\right)}\right]+\frac{\delta}{2 \sigma_{y}^{2}} \gamma_{i}\left(1-\tau_{i}\right)\left(2 \ln y-2 \mu_{0}+\delta\right) \\
& +\left(1-\gamma_{i}\right)\left(1-\tau_{i}\right) \log \left[\frac{1-\Phi\left(\frac{\ln c r-\mu_{0}}{\sigma_{y}}\right)}{1-\Phi\left(\frac{\ln c r-\left(\mu_{0}-\delta\right)}{\sigma_{y}}\right)}\right] .
\end{aligned}
$$

$$
w_{2}=\left\{\begin{array}{lll}
\frac{y_{2}}{2} e^{\frac{1}{2} \sigma_{2}^{2}+\alpha_{0}+\alpha_{1} x+\alpha_{2} \log \left(w_{1}\right)\left(1+\operatorname{erf}\left(\frac{\alpha_{0}+\alpha_{1} x+\alpha_{2} \log \left(w_{1}\right)-\log \left(c_{2}\right)+\sigma_{2}^{2}}{\sqrt{2} \sigma_{2}}\right)\right)} & \text { if } \quad y_{2} \leq c_{2} \text { and } y_{2} \leq c r_{2} \\
\frac{1}{2\left(c_{2}\right)} & \text { if } \quad c_{2}<y_{2} \text { and } c_{2} \leq c r_{2} \\
\frac{1}{\frac{1}{2} \sigma_{2}^{2}+\alpha_{0}+\alpha_{1} x+\alpha_{2} \log \left(w_{1}\right)\left(1+\operatorname{erf}\left(\frac{\alpha_{0}+\alpha_{1} x+\alpha_{2} \log \left(w_{1}\right)-\log \left(c r_{2}\right)+\sigma_{2}^{2}}{\sqrt{2} \sigma_{2}}\right)\right)} & \text { otherwise. }
\end{array}\right.
$$

Moreover, occurring of the censoring mechanism changes the likelihood function of survival time. Hence, the likelihood function associated with the $i$ th observation is defined as follows, where the probability density and the cumulative distribution functions for $y$ are shown by $f$ and $F$ and by $g$ and $G$ for $c r$, respectively:

$$
\begin{aligned}
L_{i}= & {[(1-F(c))(1-G(c))]^{\tau_{i}} \times\left[\left[f\left(y_{i}\right)(1\right.\right.} \\
& \left.\left.-G\left(y_{i}\right)\right]^{\gamma_{i}}\left[g\left(c r_{i}\right)\left(1-F\left(c r_{i}\right)\right)\right]^{\left(1-\gamma_{i}\right)}\right]^{1-\tau_{i}},
\end{aligned}
$$

in which

$$
\begin{aligned}
& \tau_{i}=\left\{\begin{array}{ll}
1 & \text { if } \quad c \leq y_{i} \text { and } c \leq c r_{i} \\
0 & \text { if } \quad c>y_{i} \text { and } c>c r_{i}
\end{array}\right. \text { and } \\
& \gamma_{i}=\left\{\begin{array}{lll}
1 & \text { if } \quad y_{i} \leq c r_{i} \\
0 & \text { if } \quad y_{i}>c r_{i} .
\end{array}\right.
\end{aligned}
$$

For the simplicity, variable $t$ is defined as $t_{i}=\min \left(y_{i}, c r_{i}, c\right)$.

Therefore, the likelihood function is written as follows:

$$
\begin{aligned}
L_{i}= & {\left[\left(1-F\left(t_{i}\right)\right)\left(1-G\left(t_{i}\right)\right)\right]^{\tau_{i}} \times\left[\left[f\left(t_{i}\right)(1\right.\right.} \\
& \left.\left.\left.-G\left(t_{i}\right)\right)\right]^{\gamma_{i}}\left[g\left(t_{i}\right)\left(1-F\left(t_{i}\right)\right)\right]^{\left(1-\gamma_{i}\right)}\right]^{1-\tau_{i}} .
\end{aligned}
$$

The CUSUM statistic regarding the presence of censoring is similar to Eq. (7). However, it is constructed based on the CUSUM score in Eq. (27) to identify the decreasing shifts. Then, it is needed to set the lower control limit shown as $\mathrm{LCL}_{3}$ and compared with the CUSUM statistic. Note that the one-sided CUSUM control chart generates a signal when this statistic is less than $\mathrm{LCL}_{3}$.

Another control scheme is EWMA control chart which was described completely. There is a critical issue that the proposed residual is not suitable in the presence of censoring, because the observations are not the actual value and the mean and variance of them would be changed. Hence, we present a new residual based on the $\mathrm{CEV}$ weights as follows:

$z_{i}^{\prime}=\frac{W_{2 i}-\mathrm{E}\left(W_{2 i}\right)}{\sqrt{\operatorname{Var}\left(W_{2 i}\right)}}$

where $E\left(W_{2 i}\right)$ and $\operatorname{Var}\left(W_{2 i}\right)$ are the mean and variance of observations. Its mean can be obtained as

$$
\begin{aligned}
E\left(W_{2}\right)= & y_{2} \times p\left(y_{2} \leq c_{2} \text { and } y_{2} \leq c r_{2}\right)+w_{c_{2}} \\
& \times p\left(c_{2} \leq y_{2} \text { and } c_{2} \leq c r_{2}\right)+w_{c r_{2}} \\
& \times p\left(c r_{2} \leq y_{2} \text { and } c r_{2} \leq c_{2}\right) .
\end{aligned}
$$


The variance of observations is computed based on the traditional definition; $\operatorname{Var}\left(W_{2}\right)=E\left(W_{2}^{2}\right)-\mathrm{E}^{2}\left(W_{2}\right)$. Finally, by calculating the probability in Eq. (29), the mean and $E\left(W_{2}^{2}\right)$ can be indicated as follows:

$$
\begin{aligned}
E\left(W_{2}^{2}\right)= & \int_{0}^{c_{2}} y_{2}^{2} \times f_{y_{2}}\left(y_{2}\right) \times\left(1-G_{c r_{2}}\left(y_{2}\right)\right) \mathrm{d}_{y_{2}} \\
& +W_{c_{2}}^{2} \times\left[1-F_{y_{2}}\left(c_{2}\right)\right] \times\left[1-G_{c r_{2}}\left(c_{2}\right)\right] \\
& +\int_{0}^{c} W_{c r_{2}}^{2} \times g_{c r_{2}}\left(c r_{2}\right) \times\left(1-F_{y_{2}}\left(c r_{2}\right)\right) \mathrm{d}_{c r_{2}} .
\end{aligned}
$$

The presented mean and variance of observations lead to long time to set the lower control limit, $\mathrm{LCL}_{4}$ by simulation. Therefore, we propose the general mean and variance. The significant advantage in applying the general mean and variance is that the censoring mechanism in the second stage does not influence on the last stage, although the simulation error to set the lower control limit would increase. In addition, the general mean and variance of quality characteristic in the third stage are fixed for each observation. The general $E\left(W_{2}\right)$ and $E\left(W_{2}^{2}\right)$, to compute the variance of observations, are indicated by the following formulas:

$$
\begin{aligned}
& \mathrm{E}\left(W_{2}\right)=\int_{0}^{c_{2}} \int_{y_{1}} \int_{x} \frac{1}{\sigma_{y_{2}} \sqrt{2 \pi}} e^{-\frac{1}{2}\left(\frac{\ln \left(y_{2}\right)-\alpha_{0}-\alpha_{1} x-\alpha \log \left(y_{1}\right)}{\sigma_{y_{2}}}\right)} \\
& \times\left[\int_{y_{2}}^{+\infty} \frac{1}{\sigma_{c r_{2}} \sqrt{2 \pi}} e^{-\frac{1}{2}\left(\frac{c r_{2}-\mu_{c r_{2}}}{\sigma_{c r_{2}}}\right)^{2}} \mathrm{~d} c r_{2}\right] \times f_{x}(x) \times f_{y_{1}}\left(y_{1}\right) \mathrm{d}_{x} \mathrm{~d}_{y_{1}} \mathrm{~d}_{y_{2}} \\
& +\int_{y_{1}} \int_{x} \frac{\frac{1}{2} e^{\frac{1}{2} \sigma_{y_{2}}^{2}+\alpha_{0}+\alpha_{1} x+\alpha_{2} \log \left(y_{1}\right)}\left(1+\operatorname{erf}\left(\frac{\alpha_{0}+\alpha_{1} x+\alpha_{2} \log \left(y_{1}\right)-\log \left(c_{2}\right)+\sigma_{y_{2}}^{2}}{\sqrt{2} \sigma_{y_{2}}}\right)\right)}{S\left(c_{2}\right)} \\
& \times\left[1-\phi_{y_{2}}\left(\frac{\ln c-\alpha_{0}-\alpha_{1} x-\alpha_{2} \log \left(y_{1}\right)}{\sigma_{y_{2}}}\right)\right] \\
& \times f_{x}(x) \times f_{y_{1}}\left(y_{1}\right) \mathrm{d}_{x} \mathrm{~d}_{y_{1}} \times\left[\int_{c_{2}}^{+\infty} \frac{1}{\sigma_{c r_{2}} \sqrt{2 \pi}} e^{-\frac{1}{2}\left(\frac{c_{r_{2}}-\mu_{c r_{2}}}{\sigma_{c r_{2}}}\right)^{2}} \mathrm{~d} c r_{2}\right] \\
& +\int_{0}^{c_{2}} \int_{y_{1}} \int_{x} \frac{1}{2} e^{\frac{1}{\sigma} \sigma_{y_{2}}^{2}+\alpha_{0}+\alpha_{1} x+\alpha_{2} \log \left(y_{1}\right)} \frac{\left(1+\operatorname{erf}\left(\frac{\alpha_{0}+\alpha_{1} x+\alpha_{2} \log \left(y_{1}\right)-\log \left(c r_{2}\right)+\sigma_{y_{2}}^{2}}{\sqrt{2} \sigma_{y_{2}}}\right)\right)}{S\left(c r_{2}\right)} \\
& \times \frac{1}{\sigma_{c r_{2}} \sqrt{2 \pi}} e^{-\frac{1}{2}\left(\frac{c_{2}-\mu_{c_{2}}}{\sigma_{c r_{2}}}\right)^{2}} \times\left[1-\phi_{y_{2}}\left(\frac{\ln c r_{2}-\alpha_{0}-\alpha_{1} x-\alpha_{2} \log \left(y_{1}\right)}{\sigma_{y_{2}}}\right)\right] \\
& \times f_{x}(x) \times f_{y_{1}}\left(y_{1}\right) \mathrm{d}_{x} \mathrm{~d}_{y_{1}} \mathrm{~d} c r_{2},
\end{aligned}
$$

and

$$
\begin{aligned}
& E\left(W_{2}^{2}\right)=\int_{0}^{c_{2}} \int_{y_{1}} \int_{x} y_{2} \times \frac{1}{\sigma_{y_{2}} \sqrt{2 \pi}} e^{-\frac{1}{2}\left(\frac{\ln \left(y_{2}\right)-\alpha_{0}-\alpha_{1} x-\alpha_{2} \log \left(y_{1}\right)}{\sigma_{y_{2}}}\right)} \\
& \times\left[\int_{y_{2}}^{+\infty} \frac{1}{\sigma_{c r_{2}} \sqrt{2 \pi}} e^{-\frac{1}{2}\left(\frac{c r_{2}-\mu_{c r_{2}}}{\sigma_{c r_{2}}}\right)^{2}} \mathrm{~d} c r_{2}\right] \times f_{x}(x) \times f_{y_{1}}\left(y_{1}\right) \mathrm{d}_{x} \mathrm{~d}_{y_{1}} \mathrm{~d}_{y_{2}}
\end{aligned}
$$

$$
\begin{aligned}
& +\int_{y_{1}} \int_{x}\left[\frac{\frac{1}{2} e^{\frac{1}{2} \sigma_{y_{2}}^{2}+\alpha_{0}+\alpha_{1} x+\alpha_{2} \log \left(y_{1}\right)}\left(1+\operatorname{erf}\left(\frac{\alpha_{0}+\alpha_{1} x+\alpha_{2} \log \left(y_{1}\right)-\log \left(c_{2}\right)+\sigma_{y_{2}}^{2}}{\sqrt{2} \sigma_{y_{2}}}\right)\right)}{S\left(c_{2}\right)}\right]^{2} \\
& \times\left[1-\phi_{y_{2}}\left(\frac{\ln c-\alpha_{0}-\alpha_{1} x-\alpha_{2} \log \left(y_{1}\right)}{\sigma_{y_{2}}}\right)\right] \\
& \times f_{x}(x) \times f_{y_{1}}\left(y_{1}\right) \mathrm{d}_{x} \mathrm{~d}_{y_{1}} \times\left[\int_{c_{2}}^{+\infty} \frac{1}{\sigma_{c r_{2}} \sqrt{2 \pi}} e^{-\frac{1}{2}\left(\frac{c_{2}-\mu_{c r_{2}}}{\sigma_{c r_{2}}}\right)^{2}} \mathrm{~d} c r_{2}\right] \\
& +\int_{0}^{c_{2}} \int_{y_{1}} \int_{x}\left[\frac{\frac{1}{2} e^{\frac{1}{2} \sigma_{y_{2}}^{2} \alpha_{0}+\alpha_{1} x+\alpha_{2} \log \left(y_{1}\right)}\left(1+\operatorname{erf}\left(\frac{\alpha_{0}+\alpha_{1} x+\alpha_{2} \log \left(y_{1}\right) \log \left(c r_{2}\right)+\sigma_{y_{2}}^{2}}{\sqrt{2} \sigma_{y_{2}}}\right)\right)}{S\left(c r_{2}\right)}\right]^{2} \\
& \times \frac{1}{\sigma_{c r_{2}} \sqrt{2 \pi}} e^{-\frac{1}{2}\left(\frac{c_{r_{2}}-\mu_{c r_{2}}}{\sigma_{c r_{2}}}\right)^{2}} \times\left[1-\phi_{y_{2}}\left(\frac{\ln c r_{2}-\alpha_{0}-\alpha_{1} x-\alpha_{2} \log \left(y_{1}\right)}{\sigma_{y_{2}}}\right)\right] \\
& \times f_{x}(x) \times f_{y_{1}}\left(y_{1}\right) \mathrm{d}_{x} \mathrm{~d}_{y_{1}} \mathrm{~d} c r_{2} .
\end{aligned}
$$

Finally, obtaining the residuals, the one-sided EWMA statistic is constructed. The EWMA control chart signals an alarm when the EWMA statistic falls below $\mathrm{LCL}_{4}$. $\mathrm{LCL}_{4}$ is obtained by simulation to achieve a desired in-control ARL.

It should be noted that a three-stage process has been studied in this paper. One may easily consider a multistage process in which the response variable is dependent on several preceding quality variables. Subsequently, the parameters of the survival regression model can be modeled in terms of all effective prior quality variables for a multistage process with $n$ stages as follows:

$\mu_{y_{n-1} \mid x, y_{1}, \ldots, y_{n-2}}=\alpha_{0}+\alpha_{1} x+\sum_{i=2}^{n-1} \alpha_{i} \log \left(y_{i-1}\right)$.

\section{Performance analysis}

This section analyzes and compares the performance of the proposed control charts to determine which monitoring method is better at detecting decreasing step shifts in the response mean or equivalently the location parameter of the lognormal distribution. It is remarkable that the simulation runs are performed to calculate ARLs with 10,000 replicates. In addition, the lower control limit for all the control charts is set through simulation to achieve the incontrol ARL of roughly 200.

The simulation studies are done with assuming these input parameters: $\lambda=0.2, \beta_{0}=1.5, \beta_{1}=-1, \alpha_{0}=2$, $\alpha_{1}=-0.5, \alpha_{2}=-0.5, x \sim N(2,1), \sigma_{y 1}=1.3$, and $\sigma_{y 2}=1$. Moreover, the presence of variable-competing risk is assumed and we aware of its occurrence which follows a normal distribution. The in-control parameters of normal distribution and also the fixed-censoring level are selected, respectively, to reach the situation in which the censoring 
Table 1 Comparison of the control charts in the absence of competing risk in the last stage

\begin{tabular}{|c|c|c|c|c|c|c|c|}
\hline \multirow[t]{2}{*}{ Control charts } & \multirow{2}{*}{$\begin{array}{l}\text { Censoring rate in the } \\
\text { second stage }(\%)\end{array}$} & \multicolumn{6}{|l|}{$\delta^{\prime}$} \\
\hline & & 0 & 0.05 & 0.1 & 0.2 & 0.5 & 1 \\
\hline \multirow[t]{4}{*}{ EWMA } & 0 & 200.53 & 144.15 & 109.04 & 65.68 & 22.86 & 9.76 \\
\hline & 20 & 200.76 & 146.43 & 110.50 & 67.04 & 23.09 & 9.77 \\
\hline & 50 & 200.35 & 152.08 & 116.81 & 73.11 & 25.65 & 10.17 \\
\hline & 80 & 200.92 & 155.42 & 122.62 & 79.74 & 28.33 & 10.62 \\
\hline \multirow[t]{4}{*}{ CUSUM } & 0 & 200.61 & 139.54 & 101.05 & 58.24 & 19.43 & 8.20 \\
\hline & 20 & 200.78 & 144.10 & 104.43 & 59.82 & 19.53 & 8.11 \\
\hline & 50 & 200.67 & 149.62 & 114.10 & 70.23 & 23.19 & 8.50 \\
\hline & 80 & 200.08 & 153.91 & 121.94 & 79.95 & 27.44 & 9.16 \\
\hline
\end{tabular}

rate becomes approximately $50 \%$ for the third-stage quality characteristic. Adopting a special value for competing risks to achieve a special censoring rate of two fixed- and variable-competing risks for both the second and third stages is described in Appendices A and B, respectively.

The ARL values of the control charts are compared; under the presence and absence of censoring in the quality characteristic of the last stage. In addition, the independency of the proposed cause-selecting control charts under no censoring scenario against the shifts in the mean quality characteristics of the first and second stages is shown in this section.

\section{Performance analysis under the absence of censoring in the last stage}

In this sub-section, the ARL of the proposed control charts is compared in the absence of both the fixed- and variablecompeting risks. Moreover, the presence of censoring in the second stage is considered to show its effect on the performance of the proposed control charts because of assuming quality characteristic of the second stage as reliability data. The results of ARL are summarized in Table 1 by considering three censoring rates of low $(20 \%)$, moderate $(50 \%)$, and high $(80 \%)$ in which the fraction of the fixed- and variable-competing risks is assumed the same.

As illustrated in Table 1, the ARL values of the CUSUM control chart are less than the ARL values of EWMA control chart under different shifts. It implies that the proposed CUSUM control chart performs better in all censoring rates in the second stage. Besides, censoring scenario in the second stage has a detrimental effect on the performance of control charts and leads to increasing the corresponding out-of-control ARL values.

\section{Performance analysis under the presence of censoring in the last stage}

The detection ability of the proposed control charts is evaluated and compared when both the fixed- and variable- competing risks take place in the last stage. Three censoring rates of 20,50 , and $80 \%$ are assumed in both the second and third stages. As discussed earlier, the proposed EWMA control chart is independent of which censoring rate happens in the second stage. Then, the ARL values are shown in Table 2.

The outcomes of performing simulation study indicate that CUSUM control chart outperforms the EWMA control chart. There is an exception that the EWMA control chart performs better in the low and moderate (20 and $50 \%$ ) censoring rates when $80 \%$ of outcomes of the second-stage quality characteristic are censored under the small shifts. In general, the performance of control charts deteriorates as the censoring rate increases.

\section{Performance analysis under mean shifts in the previous sub-processes}

In this section, we investigate that the proposed control charts are CSC through simulation. In other words, any shifts in the mean of quality characteristics in the first and second stages do not affect the ARL of the CSC control charts developed to monitor the quality characteristic in the third stage. For this purpose, the performance of the proposed control charts is studied under decreasing shifts of $E(X)-\gamma \sigma_{X}$ or $E\left(y_{1}\right)-\vartheta \sigma_{y_{1}}$ in the mean of $X$ and $Y_{1}$. Table 3 presents the ARL results in the absence of censoring with decreasing shifts, in the mean of the first- and second-stage quality characteristics.

\section{A numerical example}

To evaluate and compare the performance of the proposed monitoring procedures, a numerical example is used in this section. It is remarkable that the skein strength and fiber strength aim to reflect the product reliability in this case. We consider some scenarios to evaluate the performance of the proposed EWMA and CUSUM control charts. In the 
Table 2 Comparison of the control charts in the presence of competing risk in the last stage

\begin{tabular}{|c|c|c|c|c|c|c|c|c|}
\hline \multirow{2}{*}{$\begin{array}{l}\text { Control } \\
\text { charts }\end{array}$} & \multirow{2}{*}{$\begin{array}{l}\text { Censoring rate in } \\
\text { third stage }(\%)\end{array}$} & \multirow{2}{*}{$\begin{array}{l}\text { Censoring rate in the } \\
\text { second stage }(\%)\end{array}$} & \multicolumn{6}{|l|}{$\delta^{\prime}$} \\
\hline & & & 0 & 0.05 & 0.1 & 0.2 & 0.5 & 1 \\
\hline \multirow[t]{3}{*}{ EWMA } & 20 & 0 & 200.26 & 155.90 & 120.44 & 78.78 & 30.11 & 12. 67 \\
\hline & 50 & 0 & 200.87 & 160.32 & 127.34 & 83.82 & 34.68 & 13.83 \\
\hline & 80 & 0 & 200.02 & 169.40 & 144.09 & 104.62 & 46.66 & 19.05 \\
\hline \multirow[t]{12}{*}{ CUSUM } & 20 & 0 & 200.95 & 139.61 & 101.47 & 58.67 & 19.78 & 8.23 \\
\hline & & 20 & 200.32 & 145.75 & 105.13 & 60.70 & 20.11 & 8.29 \\
\hline & & 50 & 200.02 & 150.42 & 115.01 & 70.72 & 24.14 & 8.62 \\
\hline & & 80 & 201.46 & 157.37 & 123.70 & 80.40 & 28.37 & 9.25 \\
\hline & 50 & 0 & 199.96 & 144.25 & 104.90 & 60.85 & 20.55 & 8.38 \\
\hline & & 20 & 199.56 & 146.74 & 109.77 & 64.54 & 21.14 & 8.40 \\
\hline & & 50 & 199.78 & 151.68 & 116.95 & 71.93 & 25.26 & 8.83 \\
\hline & & 80 & 200.25 & 161.55 & 128.42 & 84.74 & 29.20 & 9.36 \\
\hline & 80 & 0 & 199.91 & 153.73 & 117.84 & 72.85 & 26.74 & 10.18 \\
\hline & & 20 & 199.95 & 154.52 & 119.60 & 75.16 & 27.14 & 10.27 \\
\hline & & 50 & 200.7 & 160.33 & 127.59 & 82.99 & 29.77 & 10.70 \\
\hline & & 80 & 200.17 & 164.77 & 131.76 & 87.02 & 32.49 & 11.19 \\
\hline
\end{tabular}

Table 3 ARLs of the CSC charts under shift in the mean of the firstand second-stage quality characteristics

\begin{tabular}{lllllll}
\hline \multicolumn{2}{l}{$E(X)-\theta \sigma_{X}$} & & & \multicolumn{3}{l}{$E\left(y_{1}\right)-\vartheta \sigma_{y_{1}}$} \\
\cline { 1 - 2 } \cline { 6 - 7 }$\theta$ & EWMA & CUSUM & & $\vartheta$ & EWMA & CUSUM \\
\hline 0 & 200.53 & 200.61 & & 200.53 & 200.61 \\
0.5 & 202.53 & 200.22 & & 0.5 & 199.81 & 199.89 \\
1 & 203.29 & 201.15 & & 1 & 203.16 & 202.06 \\
1.5 & 201.42 & 199.61 & & 1.5 & 201.05 & 199.72 \\
2 & 200.22 & 199.95 & & 202.19 & 201.66 \\
\hline
\end{tabular}

first scenario, there are no censored data in both the second and third stages. The second scenario includes high-censored data $(80 \%)$ in the second stage. In the third scenario, censoring occurs in the third stage by the high-censoring rate $(80 \%)$. In addition, the presence of censoring in both the second and third stages is considered as the last scenario.

In the illustrative example, the data are generated by the parameters which are introduced at the previous section. After generating 10 in-control samples, the data are generated under the out-of-control status. This status is occurred by a decreasing shift in the mean of quality characteristic in the third stage which is equal to 0.2.

The CUSUM and EWMA control charts for the data set are shown in Fig. 2. The lower control limit of the EWMA control chart is -0.4507 , and the lower control limit of the CUSUM control chart is -2.8 . As shown in this figure, the CUSUM control chart performs better than EWMA control chart.

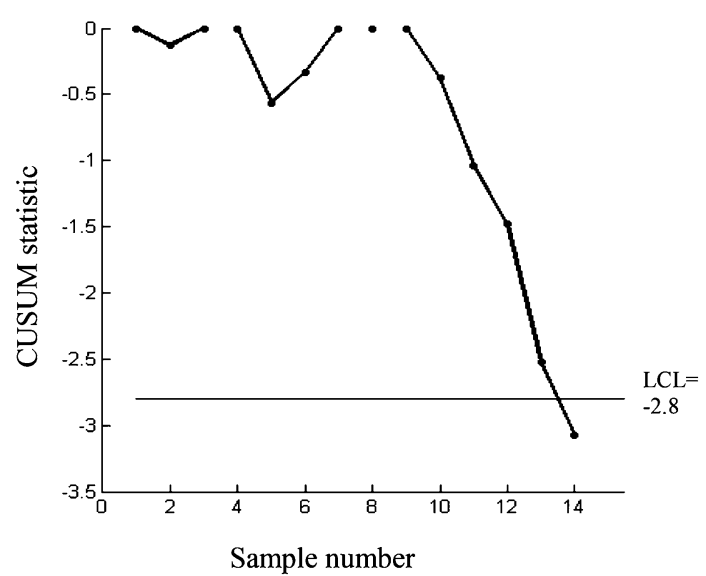

(a) Lower one-sided CUSUM control chart

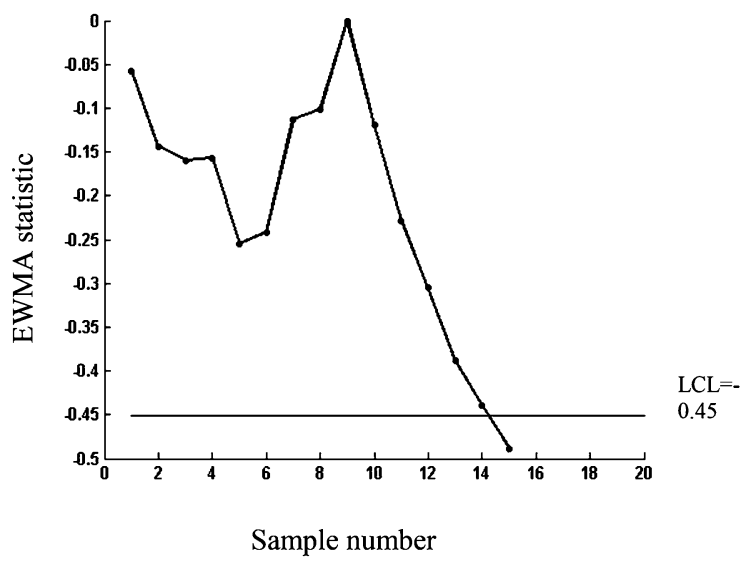

(b) Lower one-sided EWMA control chart

Fig. 2 Proposed control charts under the absence of censoring 


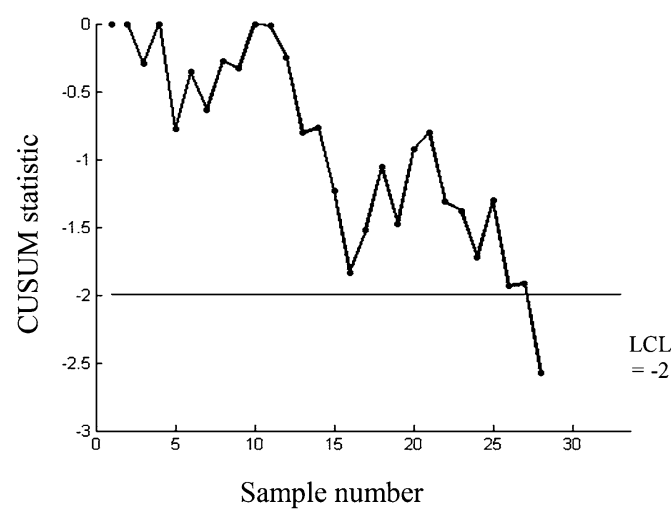

(a) Lower one-sided CUSUM control chart in the presence of $80 \%$ rate censoring

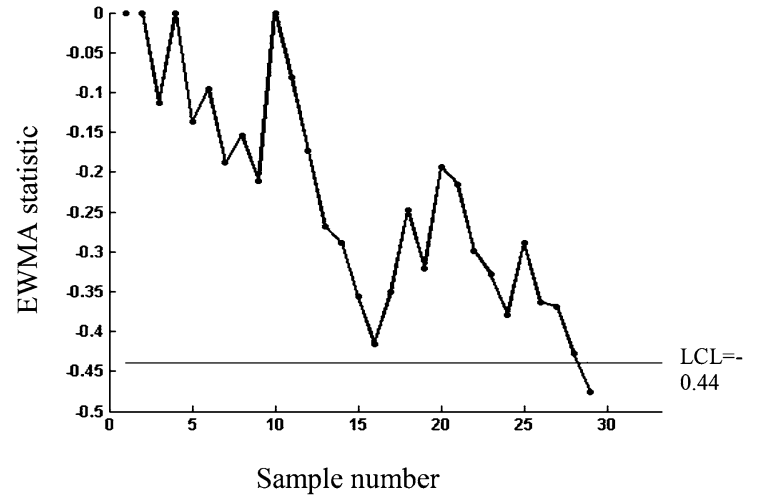

(b) Lower one-sided EWMA control chart in the presence of $80 \%$ censoring rate

Fig. 3 Proposed control charts under the presence of high-censoring rate in the second stage

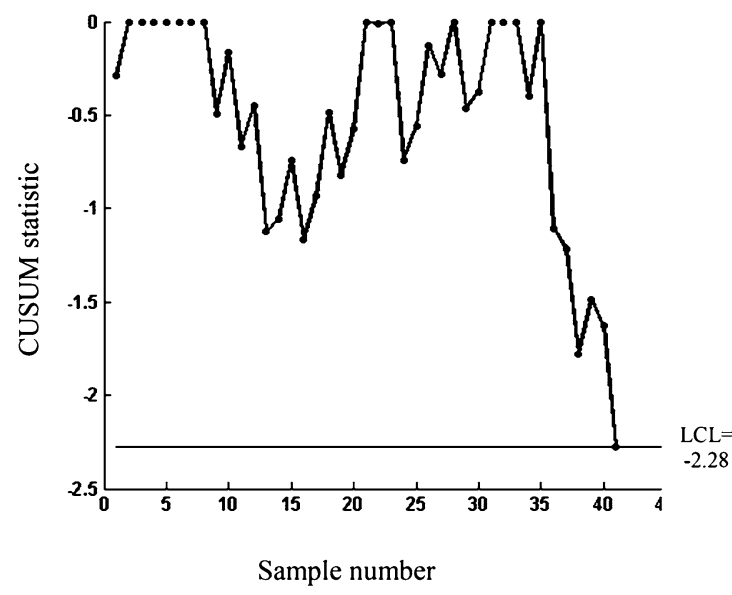

(a) Lower one-sided CUSUM control chart in the presence of $80 \%$ rate censoring

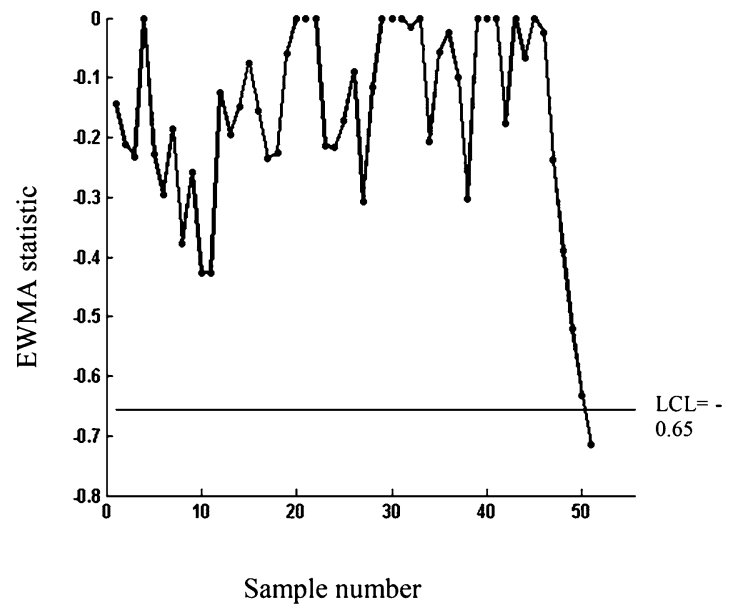

(b) Lower one-sided EWMA control chart in the presence of $80 \%$ censoring rate

Fig. 4 Proposed control charts under the presence of high-censoring rate in the third stage

As illustrated in Fig. 3, the CUSUM control chart outperforms the EWMA control chart in detecting decreasing shift in the presence of censoring in the second stage. It should be noted that the values of the fixed- and meanvariable-competing risks are obtained by solving the equations in Appendix A, i.e., $c=0.1583$ and $\mu_{c r}=0.1685$. In addition, the LCL of the EWMA and the LCL of the CUSUM control charts are -0.44 and -2 , respectively.

Figure 4 shows the CUSUM and EWMA control charts under the presence of high-censoring rate $(80 \%)$ in the third stage, respectively. In this case, we have $c=3.8901$, $\mu_{c r}=3.9883, \mathrm{LCL}=-2.28$, and $\mathrm{LCL}=-0.6568$. In addition, the mean and variance of observations are obtained generally based on Eqs. (31) and (32), whose values are equal to 6.7356 and 31.7678 , respectively. It is clear that the CUSUM control chart performs better than the EWMA control chart in this case.
Figure 5 shows the control charts under the last scenario when the data are censored in moderate rate $(50 \%)$ of the last stage and the high rate $(80 \%)$ of the second stage. The fixed and mean variable completing risks of the second stage are similar to the previous cases, and for the third stage, its values are 3.9 and 3.99, respectively. In addition, the mean and variance of observations under the moderate censoring rate are equal to 7.1075 and 71.6985 , respectively. Moreover, the lower control limit of the EWMA and the lower control limit of the CUSUM control charts are -0.68 and -1.9 , respectively. The figure implies that the performance of EWMA control chart is better than the CUSUM control chart in this situation.

By comparing the figures, we conclude that as the censoring rate decreases, the performance of control charts deteriorates. In addition, the effect of censoring rate on the performance of both EWMA and CUSUM control charts in the third stage is more than the censoring rate in the second 


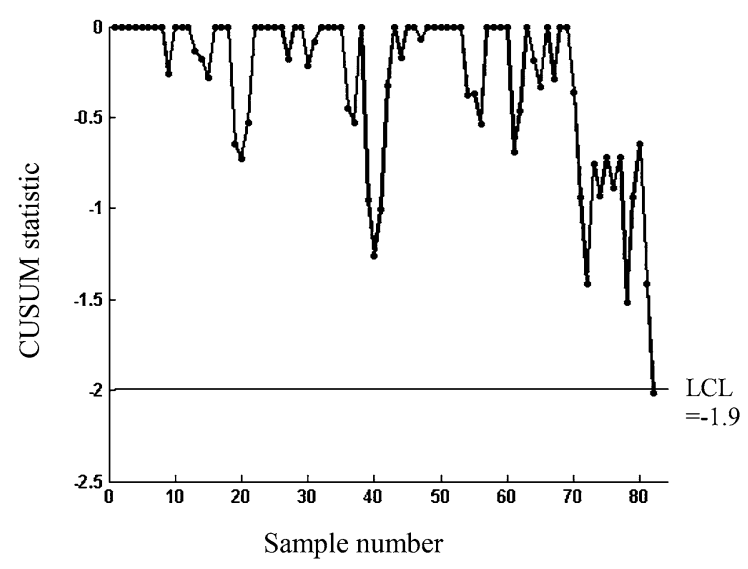

(a) Lower one-sided CUSUM control chart in the presence of $80 \%$ rate censoring in the second stage and $50 \%$ rate censoring in the third stage

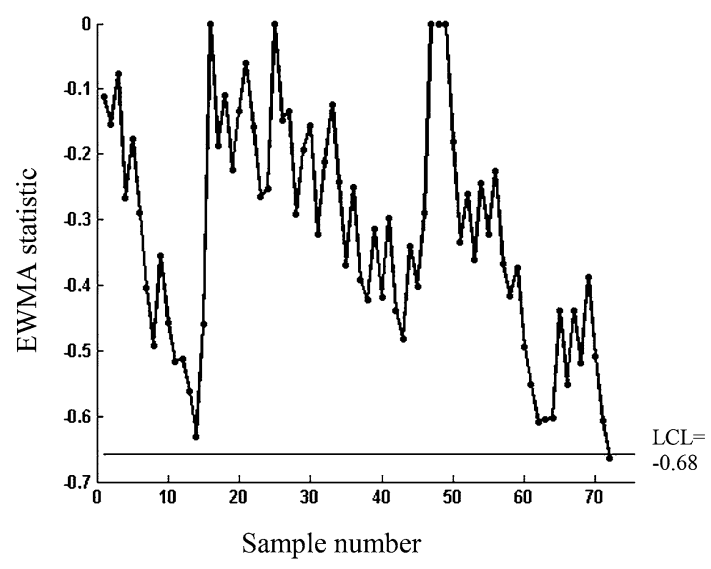

(b) Lower one-sided EWMA control chart in the presence of $80 \%$ rate censoring in the second stage and $50 \%$ rate censoring in the third stage

Fig. 5 Proposed control charts under the presence of high-censoring rate in the second and third stages

stage. It means that the control charts perform better when the censoring mechanism ( $80 \%$ ) is occurred in the second stage instead of the third stage.

\section{Conclusion and future researches}

In this paper, a three-stage process with reliability-related quality characteristic in the second and third stages was considered. We assumed that the quality characteristics of the second and third stages follow lognormal distribution due to its vast application in real world. In addition, censoring under both fixed- and variable-competing risks was considered in this study. The AFT model, which is the most widely used among the survival regression models, was considered to relate the quality characteristics in three stages. Then, we designed the EWMA and CUSUM causeselecting control charts based on this model and conditional expected value to alleviate censoring mechanism. The performances of monitoring schemes were investigated by the ARL values. The results of simulation study confirmed that the censoring scenario in each stage deteriorates the performance of the proposed control charts. Moreover, the CUSUM control chart was usually better than the EWMA control chart to detect shifts. The EWMA control chart performs better just in the high-censoring rate of the third stage when the censoring rate of the second stage is 20 or $50 \%$ simultaneously. Finally, it was shown that the monitoring procedures are independent against changing the mean shifts of the preceding stages. Moreover, an illustrative example was provided to show the performance of control charts. It is clear that the censoring would affect the performance of the control charts and leads to deteriorating the performance of the control charts. It was also shown that the effect of the censoring on the performance of the control charts is larger when the censoring happens in the third stage rather than the previous stages. The authors suggest using the proposed methods of this paper in a real case as a future research. Furthermore, in this paper, we considered one quality characteristic in each stage. However, sometimes, this assumption violates, and there is more than one quality characteristic in each stage. Therefore, developing control charts for monitoring the multivariate lognormal quality characteristics in a three-stage process could be considered as a future research. In addition, the quality characteristics in different stages may follow different distributions. This case can also be considered as a fruitful area for future researches.

Acknowledgments The authors are thankful to the respectful anonymous referees for precious comments which led to improvement in the paper.

Open Access This article is distributed under the terms of the Creative Commons Attribution 4.0 International License (http://crea tivecommons.org/licenses/by/4.0/), which permits unrestricted use, distribution, and reproduction in any medium, provided you give appropriate credit to the original author(s) and the source, provide a link to the Creative Commons license, and indicate if changes were made.

\section{Appendix A: Determining the values of the fixed- competing risk and the mean of variable- competing risk corresponding to the quality characteristic of the second stage to achieve a specified percentage of censoring}

In a three-stage process assume that the quality characteristics of the first, second, and third stages follow normal, lognormal, and lognormal distributions, respectively, shown by $f_{x}, f_{y_{1}}$, and $f_{y_{2}}$, and the variable-competing risk of 
the second stage follows normal distribution. To determine the accurate values of the fixed-censoring level and the mean of variable-competing risk of the second stage, the following equations should be solved:

$\left\{\begin{array}{l}P(\text { Censoringduetothefixedcompetingrisk })=a \\ P(\text { Censoringduetothevariablecompetingrisk })=b,\end{array}\right.$

where these formulas are computed as follows:

$P$ (Censoring due to the fixed competing risk)

$$
\begin{aligned}
& =P\left(y_{1}>c_{1} \cap c r_{1}>c_{1}\right)=P\left(y_{1}>c_{1}\right) \times P\left(c r_{1}>c_{1}\right) \\
& =\int_{x}\left[1-F_{y_{1}}\left(c_{1}\right)\right] f_{x}(x) \mathrm{d} x \times\left[1-F_{c r_{1}}\left(c_{1}\right)\right] \\
& =\int_{x}\left[1-\phi_{y_{1}}\left(\frac{\log c_{1}-\beta_{0}-\beta_{1} x}{\sigma_{y_{1}}}\right)\right] \frac{1}{\sigma_{x} \sqrt{2 \pi}} e^{-\frac{1}{2}\left(\frac{x-\mu_{x}}{\sigma_{x}}\right)^{2}} \mathrm{~d} x \\
& \times\left[\int_{c_{1}}^{+\infty} \frac{1}{\sigma_{c r_{1}} \sqrt{2 \pi}} e^{-\frac{1}{2}\left(\frac{c r-\mu_{c r_{1}}}{\sigma_{c r_{1}}}\right)^{2}} \mathrm{~d} c r_{1}\right],
\end{aligned}
$$

and

$P$ (Censoring due to the variable competing risk)

$$
\begin{aligned}
= & P\left(y_{1}>c r_{1} \cap c r_{1}<c_{1}\right) \\
= & \int_{0}^{c_{1}} \int_{c_{1}}^{r_{1}} f_{y_{1}, c r_{1}}\left(y_{1}, c r_{1}\right) \mathrm{d} c r_{1} \mathrm{~d} y_{1}+\int_{c_{1}}^{+\infty} \int_{0}^{c_{1}} f_{y_{1}, c r_{1}}\left(y_{1}, c r_{1}\right) \mathrm{d} c r_{1} \mathrm{~d} y_{1} \\
= & \int_{0}^{c_{1}} \int_{c_{1}}^{r_{r_{1}}} f_{y_{1}, c r_{1}}\left(y_{1}, c r_{1}\right) \mathrm{d} c r_{1} \mathrm{~d} y_{1}+\int_{c_{1}}^{+\infty} \int_{0}^{c_{1}} f_{y_{1}, c r_{1}}\left(y_{1}, c r_{1}\right) \mathrm{d} c r_{1} \mathrm{~d} y_{1} \\
= & \int_{x} \int_{0}^{c_{1}}\left[\phi_{y_{1}}\left(\frac{\log c_{1}-\beta_{0}-\beta_{1} x}{\sigma_{y_{1}}}\right)-\phi_{y_{1}}\left(\frac{\log c r_{1}-\beta_{0}-\beta_{1} x}{\sigma_{y_{1}}}\right)\right] \\
& \times \frac{1}{\sigma_{c r_{1}} \sqrt{2 \pi}} e^{-\frac{1}{2}\left(\frac{c r_{1}-c_{c_{1}}}{\sigma_{c r_{1}}}\right)^{2}} \times \frac{1}{\sigma_{x} \sqrt{2 \pi}} e^{-\frac{1}{2}\left(\frac{x-\mu_{x}}{\sigma_{x}}\right)^{2}} \mathrm{~d} c r_{1} \mathrm{~d} x \\
& +\left[1-\int_{c_{1}}^{+\infty} \frac{1}{\sigma_{c r_{1}} \sqrt{2 \pi}} e^{-\frac{1}{2}} \frac{\left(\frac{c r-\mu_{c_{1}}}{\sigma_{c_{1}}}\right)^{2}}{2}\right] \\
& \times \int_{x}\left[1-\phi_{y_{1}}\left(\frac{\log c_{1}-\beta_{0}-\beta_{1} x}{\sigma_{y_{1}}}\right)\right] \frac{1}{\sigma_{x} \sqrt{2 \pi}} e^{-\frac{1}{2}\left(\frac{x-\mu_{1}}{\sigma_{x}}\right)^{2}} \mathrm{~d} x .
\end{aligned}
$$

For example, if it is aimed to have a total $50 \%$ censored observations with the equal censoring rate of the fixed- and variable-competing risks, we can derive the following equations by replacing 0.25 instead of a and b in Eq. (34) as follows:
$\{P($ Censoring due to the fixed competing risk $)=0.25$

$\{P($ Censoring due to the variable competing risk $)=0.25$.

$c_{1}=0.7378$ and $\mu_{c r_{1}}=0.8698$ are obtained by solving the previous equations.

\section{Appendix B: Determining the values of fixed- censoring level and the mean of variable- competing risk to reach a specified percentage of censoring corresponding to the quality characteristic of the third stage}

In this Appendix, we calculate the accurate values of the fixed-competing risk and the mean of the variablecompeting risk of the reliability-related quality characteristic in the third stage. Therefore, all assumptions are similar to Appendix A that the quality characteristics of the first, second, and third stages follow normal, lognormal, and lognormal distributions, respectively (shown by $f_{x}, f_{y_{1}}$, and $f_{y_{2}}$ ) in a three-stage process. The fixedcompeting risk value, $c_{2}$, and the mean of the variablecompeting risk are normally distributed (denoted by $f_{c r 2}$ ). These variables are obtained by solving the following equations:

$\left\{\begin{array}{l}P(\text { Censoring due to the fixed competing risk })=a \\ P(\text { Censoring due to the variable competing risk })=b .\end{array}\right.$

Subsequently, those equations are given by the following equations:

$P$ (Censoring due to the fixed competing risk)

$$
\begin{aligned}
= & P\left(y_{2}>c_{2} \cap c r_{2}>c_{2}\right) \\
= & P\left(y_{2}>c_{2}\right) \times P\left(c r_{2}>c_{2}\right) \\
= & \int_{y_{1}} \int_{x}\left[1-F_{y_{2}}\left(c_{2}\right)\right] f_{x}(x) f_{y_{1}}\left(y_{1}\right) d x d y_{1} \times\left[1-F_{c r_{2}}\left(c_{2}\right)\right] \\
= & \int_{y_{1}} \int_{x}\left[1-\phi_{y_{2}}\left(\frac{\log c_{2}-\alpha_{0}-\alpha_{1} x_{i}-\alpha_{2} \log \left(y_{1 i}\right)}{\sigma_{y_{2}}}\right)\right] \\
& \times f_{x}(x) f_{y_{1}}\left(y_{1}\right) \mathrm{d} x \mathrm{~d} y_{1} \times\left[\int_{c_{2}}^{+\infty} \frac{1}{\sigma_{c r_{2}} \sqrt{2 \pi}} e^{-\frac{1}{2}\left(\frac{c r_{2}-\mu_{c r_{2}}}{\sigma c c_{2}}\right)^{2}} \mathrm{~d} c r_{2}\right],
\end{aligned}
$$

and 
$P($ Censoring due to the variable competing risk $)=P\left(y_{2}>c r_{2} \cap c r_{2}<c_{2}\right)$

$$
\begin{aligned}
= & \int_{0}^{c_{2}} \int_{0}^{y_{2}} f_{y_{2}, c r_{2}}\left(y_{2}, c r_{2}\right) \mathrm{d} c r_{2} \mathrm{~d} y_{2}+\int_{c_{2}}^{+\infty} \int_{0}^{c_{2}} f_{y_{2}, c r_{2}}\left(y_{2}, c r_{2}\right) \mathrm{d} c r_{2} \mathrm{~d} y_{2} \\
= & \int_{0}^{c_{2}} \int_{c_{2}}^{c r_{2}} f_{y_{2}, c r_{2}}\left(y_{2}, c r_{2}\right) \mathrm{d} c r_{2} \mathrm{~d} y_{2}+\int_{c_{2}}^{+\infty} \int_{0}^{c_{2}} f_{y_{2}, c r_{2}}\left(y_{2}, c r_{2}\right) \mathrm{d} c r_{2} \mathrm{~d} y_{2} \\
= & \int_{y_{1}} \int_{x}^{c_{2}}\left[\phi_{y_{2}}\left(\frac{\log c_{2}-\alpha_{0}-\alpha_{1} x_{i}-\alpha_{2} \log \left(y_{1 i}\right)}{\sigma_{y_{2}}}\right)-\phi_{y_{2}}\left(\frac{\log c r_{2}-\alpha_{0}-\alpha_{1} x_{i}-\alpha_{2} \log \left(y_{1 i}\right)}{\sigma_{y_{2}}}\right)\right] \\
& \times \frac{1}{\sigma_{c r_{2}} \sqrt{2 \pi}} e^{-\frac{1}{2}\left(\frac{c r_{2}-\mu_{c r_{2}}}{\sigma_{c r_{2}}}\right)^{2} \times \frac{1}{\sigma_{x} \sqrt{2 \pi}} e^{-\frac{1}{2}\left(\frac{x-\mu_{x}}{\sigma_{x}}\right)^{2}} \times \frac{1}{\sqrt{2 \pi} \sigma_{y_{2}} y_{2}} e^{\left.-\left(\frac{\log y_{2}-\beta_{0}-\beta_{1} x}{\sigma_{y_{2}}}\right)^{2}\right)} \mathrm{d} c r_{2} \mathrm{~d} x \mathrm{~d} y_{1}} \\
& +\left[1-\int_{c_{2}}^{+\infty} \frac{1}{\sigma_{c r_{2}} \sqrt{2 \pi}} e^{-\frac{1}{2}\left(\frac{c r_{2}-\mu_{c r_{2}}}{\sigma_{c r_{2}}}\right)^{2}}\right] \times \int_{y_{1}} \int_{x} \phi_{y_{2}}\left(\frac{\log c_{2}-\alpha_{0}-\alpha_{1} x_{i}-\alpha_{2} \log \left(y_{1 i}\right)}{\sigma_{y_{2}}}\right) \times \frac{1}{\sigma_{x} \sqrt{2 \pi}} e^{-\frac{1}{2}\left(\frac{x-\mu_{x}}{\sigma_{x}}\right)^{2}} \\
& \times \frac{1}{\sqrt{2 \pi} \sigma_{y_{2}} y_{2}} e^{-\left(\frac{1}{2}\left(\frac{\log y_{2}-\beta_{0}-\beta_{1} x}{\sigma_{y_{2}}}\right)^{2}\right)} \mathrm{d} x \mathrm{~d} y_{1} .
\end{aligned}
$$

As mentioned in Appendix A, if we would like to have a total of $50 \%$ censored data with equal censoring rate of the fixed- and variable-competing risks, for instance, Eq. (38) would be as follows:

$\{P($ Censoring due to the fixed competing risk $)=0.25$

$\{P($ Censoring due to the variable competing risk $)=0.25$.

After solving the above equations, $c_{2}=3.8901$ and $\mu_{c r_{2}}=$ 3.9883 are obtained.

\section{References}

Akhundjanov SH, Pascual F (2015) Moving range EWMA control charts for monitoring the Weibull shape parameter. J Stat Comput Simul 85(9):1864-1882

Asadzadeh Sh, Aghaie A (2012) Improving the product reliability in multistage manufacturing and service operations. Qual Reliab Eng Int 28(4):397-407

Asadzadeh Sh, Aghaie A, Niaki STA (2013) AFT regression-adjusted monitoring of reliability data in cascade processes. Qual Quant 47(6):3349-3362

Asadzadeh S, Aghaie A, Shahriari H, Niaki STA (2014a) The application of proportional hazards and frailty models to multistage processes surveillance. Int J Adv Manuf Technol 74(1-4):461-470

Asadzadeh Sh, Aghaie A, Shahriari H (2014b) Using frailty models to account for heterogeneity in multistage manufacturing and service processes. Qual Quant 48(2):593-604
Batson RG, Jeong Y, Fonseca DJ, Ray PS (2006) Control charts for monitoring field failure data. Qual Reliab Eng Int 22(7):733-755

Jearkpaporn D, Montgomery DC, Runger GC, Borror CM (2003) Process monitoring for correlated gamma distributed data using generalized-linear model-based control charts. Qual Reliab Eng Int 19(6):477-491

Jearkpaporn D, Borror CM, Runger GC, Montgomery DC (2007) Process monitoring for mean shifts for multiple stage processes. Int J Prod Res 45(23):5547-5570

Lawless JF (2003) Statistical models and methods for lifetime data. Wiley, New York

Li ZH, Kong ZH (2015) A generalized procedure for monitoring right-censored failure time data. Qual Reliab Eng Int 31(4):695-705

Li Z, Zhou S, Sievenpiper C, Choubey S (2012) Statistical monitoring of time-to-failure data using rank tests. Qual Reliab Eng Int 28(3):321-333

Lu W, Tsai T (2008) Exponentially weighted moving average control chart for gamma distribution with type I censoring. In: Proceedings of the 3rd international conference on innovative computing information and control, pp 128-131

Meeker WQ, Escobar LA (1998) Statistical methods for reliability data. Wiley, New York

Nichols MD, Padgett WJ (2006) A bootstrap control chart for Weibull percentile. Qual Reliab Eng Int 22(2):141-151

Padgett WJ, Spurrier JD (1990) Shewhart-type charts for percentiles of strength distributions. J Qual Technol 22(4):283-288

Page ES (1954) Continuous inspection schemes. Biometrika 41:100-115

Shafae M, Dickinson R, Woodall W, Camelio J (2014) Cumulative sum control charts for monitoring Weibull-distributed time between events. Qual Reliab Eng Int 31(5):839-849

Shamsuzzaman M, Xie M, Goh TN, Zhang HY (2009) Integerated control chart system for time between-events monitoring in a 
multistage manufacturing system. Int $\mathbf{J}$ Adv Manuf Technol 40(3-4):373-381

Skinner KR, Montgomery DC, Runger GC (2003) Process monitoring for multiple count data using generalized linear model-based control charts. Int J Prod Res 41(6):167-1180

Steiner SH, MacKay RJ (2000) Monitoring processes with highly censored data. J Qual Technol 32(3):199-208

Steiner SH, MacKay RJ (2001) Monitoring processes with data censored owing to competing risks by using exponentially weighted moving average control charts. App Stat 50:293-302

Surucu B, Sazak HS (2009) Monitoring reliability for a threeparameter Weibull distribution. Reliab Eng Syst Saf 94(2):503-508

Tsung F, Li Y, Jin M (2008) Statistical process control for multistage manufacturing and service operations: a review and some extensions. Int J Serv Oper Inf 3(2):191-204

Wade MR, Woodall WH (1993) A review and analysis of causeselecting control charts. J Qual Technol 25(3):161-169

Xiang L, Tsung F (2008) Statistical monitoring of multi-stage processes based on engineering models. IIE Trans 40(10):957-970

Xie M, Goh TN, Ranjan P (2002) Some effective control chart procedures for reliability monitoring. Reliab Eng Syst Saf 77(2): $143-150$

Yang CM (1999) Economic process management and its application on bank industry. Qual Quant 33(4):381-394

Zhang GX (1984) A new type of control charts and theory of diagnosis with control charts. In: World quality congress transactions. Milwaukee, WI: American Society for Quality Control, 3, pp 175-185

Zhang L, Chen G (2004) EWMA charts for monitoring the mean of censored Weibull lifetimes. J Qual Technol 36(3):321-328

Zhang HY, Shamsuzzaman M, Xie M, Goh TN (2011a) Design and application of exponential chart for monitoring time-betweenevents data under random process shift. Int $\mathrm{J}$ Adv Manuf Technol 57(9-12):849-857

Zhang HY, Xie M, Goh TN, Shamsuzzaman M (2011b) Economic design of time-between-events control chart system. Comput Ind Eng 60(4):485-492

Zhang M, Xu Y, He Z, Hou X (2015) The effect of estimation error on risk- adjusted survival time cusum chart performance. Qual Reliab Eng Int. doi:10.1002/qre.1849
Zhou C, Chinnam RB, Korostelev A (2012) Hazard rate models for early detection of reliability problems using information from warranty databases and upstream supply chain. Int J Prod Econ 139(1):180-195

Azam Goodarzi received her BS degree in Industrial Engineering from Alzahra University in Tehran. In addition, she holds an MS degree in Industrial Engineering from Shahed University in Tehran in 2014. Her research interests are Statistical Quality Control, Reliability, and Survival Data Analysis.

Amirhossein Amiri is an Associate Professor at Shahed University in Iran. He holds a BS, MS, and Ph.D. in Industrial Engineering from the Khajeh Nasir University of Technology, Iran University of Science and Technology, and Tarbiat Modares University in Iran, respectively. He is now a head of Industrial Engineering Department at Shahed University in Iran and a member of the Iranian Statistical Association. His research interests are statistical quality control, profile monitoring, and Six Sigma. He has published many papers in the area of statistical process control in high-quality international journals, such as Quality and Reliability Engineering International, Communications in Statistics, Computers and Industrial Engineering, Journal of Statistical Computation and Simulation, Soft Computing, and so on. He has also published a book with John Wiley and Sons in 2011 entitled the Statistical Analysis of Profile Monitoring.

Shervin Asadzadeh is an Assistant Professor in Islamic Azad University, North Tehran Branch. He holds his Ph.D. in Industrial Engineering from the K.N. Toosi University of Technology. He has been teaching Statistical Methods, Design of Experiments, Time Series Analysis, Statistical Quality Control, Quality Management and Productivity, and Engineering Statistics at the K.N. Toosi University of Technology, Allameh Tabataba'i University, and Islamic Azad University. His primary research interests include Statistical Quality Control, Reliability, Survival Data Analysis, Applied Statistics, Robust Statistics, and Simulation. He is a member of National Elites Foundation of Iran, Iranian Institute of Industrial Engineering, and Iranian Statistical Society. 